\title{
Optical Fiber Fresnel Reflection Sensor for Direct Detection of the Solid-Liquid Phase Change in N-Octadecane
}

Wei Han

Technological University Dublin, wei.han@tudublin.ie

Marek Rebow

Technological University Dublin, marek.rebow@tudublin.ie

Dejun Liu

Technological University Dublin, dejun.liu@tudublin.ie

See next page for additional authors

Follow this and additional works at: https://arrow.tudublin.ie/engscheleart2

Part of the Electrical and Electronics Commons

\section{Recommended Citation}

Han, W., Rebow, M. (2018). Optical Fiber Fresnel Reflection Sensor for Direct Detection of the Solid-Liquid Phase Change in N-Octadecane. Measurement Science and Technology, vol. 29, no. 12 doi:10.1088/

1361-6501/aaeabb

This Article is brought to you for free and open access by the School of Electrical and Electronic Engineering at ARROW@TU Dublin. It has been accepted for inclusion in Articles by an authorized administrator of ARROW@TU Dublin. For more information, please contact arrow.admin@tudublin.ie, aisling.coyne@tudublin.ie, gerard.connolly@tudublin.ie.

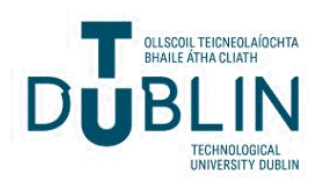


Authors

Wei Han, Marek Rebow, Dejun Liu, Yuliya Semenova, and Qiang Wu Prof.

This article is available at ARROW@TU Dublin: https://arrow.tudublin.ie/engscheleart2/190 


\title{
Optical fiber Fresnel reflection sensor for direct detection of the solid-liquid phase change in n-octadecane
}

\author{
Wei Han ${ }^{1} \oplus$, Marek Rebow ${ }^{2}$, Dejun Liu ${ }^{1}$, Gerald Farrell ${ }^{1}$, Yuliya Semenova ${ }^{1}$ \\ and Qiang $\mathbf{W u}^{3}$ (1) \\ ${ }^{1}$ Photonics Research Centre, Dublin Institute of Technology, Kevin Street, Dublin 8, Ireland \\ 2 College of Engineering and Built environment, Dublin Institute of Technology, Bolton Street, Dublin 1, \\ Ireland \\ 3 Department of Mathematics, Physics and Electrical Engineering, Northumbria University, Newcastle \\ Upon Tyne, NE1 8ST, United Kingdom \\ E-mail: d14125980@mydit.ie
}

Received 19 June 2018, revised 8 October 2018

Accepted for publication 23 October 2018

Published 16 November 2018

\begin{abstract}
An all optical fiber Fresnel reflection sensor for detecting the solid-liquid phase change in n-octadecane is proposed and experimentally demonstrated. The sensor probe consists of a single-mode fiber with a cleaved end which is immersed in the material sample under test. As the n-octadecane changes from solid to liquid or from liquid to solid, its refractive index (RI) changes resulting in changes of the light power ratio reflected by the probe. Since the RI of n-octadecane during its phase transition changes discontinuously, inflection in the reflected light power ratio dependence indicates the phase change of the sample in the vicinity of the sensor probe. The results of this work suggest that such a simple optical fiber sensor can be used for detection of liquid-solid phase changes in other materials with similar to n-octadecane thermo-optic properties.
\end{abstract}

Keywords: phase change material, optical fiber sensor, n-octadecane, supercooling

(Some figures may appear in colour only in the online journal)

\section{Introduction}

Due to the ability to absorb or release large amounts of energy during melting or solidification, phase change materials (PCMs) play an important role in practical thermal energy storage applications [1]. To achieve better energy efficiency for the energy storage process, it is necessary to be able to accurately detect the phase changes in the PCMs. The thermocouple is a simple temperature sensor that allows for indirect detection of the phase changes by measuring the temperature of the PCM. It has the advantages of simplicity and low cost, but also has some limitations. For example, due to its high thermal conductivity, the thermocouple can act as a sink or source when heat is transferred to the material by the thermocouple wire immersed into a sample, which may affect the phase state in its vicinity and thus cause errors in detection
[2]. Moreover, in the occurrence of supercooling (when a PCM remains in a liquid phase when the temperature is lower than its freezing point), the information provided by the thermocouple in relation to material's phase is not accurate.

Therefore in practice direct detection of the phase changes in PCMs is preferred. In the case of optically transparent materials, direct observation of the solid and liquid interface is possible [3]; but this technique is not applicable in opaque materials. X-ray tomography and radiography allow visualizing solid-liquid interfaces regardless of optical transparency [4] since both methods detect changes in the material density associated with the phase changes, but both methods are laboratory-based and high cost, and besides both of them potentially involve exposure of human personnel to radiation. Most materials show changes in electrical resistance upon melting, and thus resistance diagnostics has also been used for 


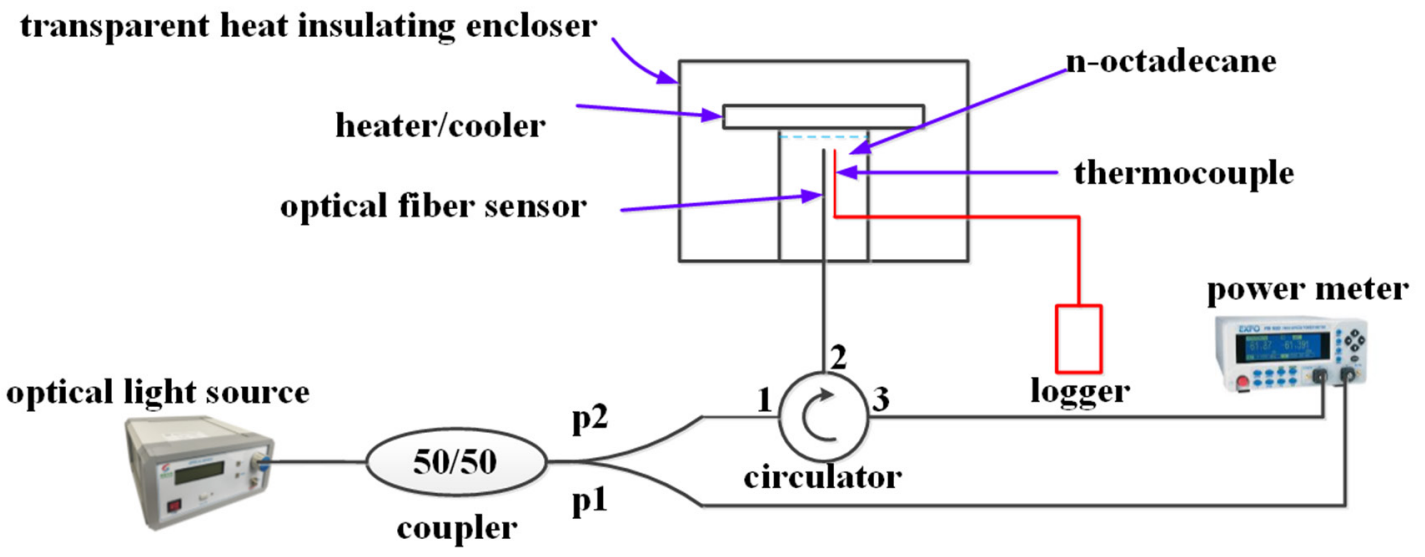

Figure 1. Schematic diagram of the experimental setup.

detecting the phase change [5]. This measurement technique involves letting electric current flow through the sample during its phase transition and measuring the changes in resistance. It is a safe measurement technique, compared with the $\mathrm{x}$-ray, but it also has the disadvantage of complexity. Differential scanning calorimetry (DSC) can be applied to characterise PCMs, and it also can be used to determine the phase state within a very small bulk material sample [6]. However, DSC does not allow the determination of the phase state at specific points within the sample's volume, and thus is unsuitable for energy storage applications which involve large PCM volumes.

Optical fiber sensors have the advantages of small size, high accuracy, immunity to electromagnetic interference, remote sensing capability and chemically passive nature. As a result, optical fiber sensors are widely used for measurements of temperature [7], strain [8] and refractive index (RI) [9]. Several previous reports illustrated that optical fiber sensors can be successfully applied for the detection of the phase change. For example, Arnon et al [10] developed a fiberoptic evanescent wave spectroscopic method for detecting the solid-liquid phase changes in water based on the changes of the sample's absorbance. However the technique is relatively complex and requires the use of special silver halide fibers. Chai et al [11] used a combination of coating-stripped and coated fiber Bragg grating (FBG) sensors to monitor the solid-liquid and liquid-gas phase transitions in water. Their proposed technique requires a minimum of two FBG sensors, whose different positions within the sample may result in errors. Mani et al [12] reported a Fresnel reflection fiber sensor for monitoring the crystallization of water and aqueous solution of $\mathrm{NaCl}$ by detecting the changes of the reflected power ratio from the probe. The main focus of their work was on studies of crystallization of distilled water under supercooling conditions as it was subjected to rapid cooling using liquid nitrogen.

In this paper we propose and experimentally demonstrate a novel method for in situ detection of the solid-liquid and liquid-solid phase changes in n-octadecane, based on Fresnel reflection in a fiber sensor probe. It is important to note that the sensing scheme proposed offers a reliable means to sense phase change in a situation where traditional optical fiber sensing methods fail to detect phase change due to the occurrence of supercooling.

The sensor probe is fabricated from a single-mode fiber with a cleaved end, and the phase change is detected by monitoring the change of the optical power ratio reflected back from the sensor probe immersed in the material sample. $\mathrm{N}$-octadecane is a popular PCM (C18H38 n-alkane material), whose thermal characteristics make it attractive for a number of applications, including thermal control in a spacecraft [13], in comfort clothing, to maintain the appropriate temperature close to that of human skin [14] and in solar thermoelectric generators [15]. In this work n-octadecane was chosen for the proof of principle demonstration of the proposed method because the material's crystallization occurs over a narrow range of temperatures, with high degree of repeatability. In addition, the closeness of the freezing point to room temperature allows for a simpler experimental set up. Moreover, the material is transparent in liquid phase and opaque in solid phase, which makes it possible to observe the phase change in the vicinity of the probe for comparison.

\section{Experimental setup and operating principle}

The experimental setup for demonstration of the proposed method is shown in figure 1 . The sensor system consists of a $3 \mathrm{~dB}$ coupler, whose input port is connected to a broadband source (S5FC1021S, Thorlabs; wavelength range: 1200$1660 \mathrm{~nm}$ ), one of the outputs of the coupler is connected to one of the channels of the optical power meter $(4100, \mathrm{dBm}$ Optics) and another output is connected with the Port 1 of the optical circulator. Ports 2 and 3 of the optical circulator are connected to the sensor probe and the second channel of the optical power meter respectively. The sensor probe is made of a standard single-mode fiber with a cleaved end immersed into the sample of n-octadecane. The coating is removed from a small area near the end of the fiber and this area of the fiber marked with red ink. The n-octadecane sample is placed in a transparent glass container with a diameter of $2 \mathrm{~cm}$ and a height of $8 \mathrm{~cm}$. Since the liquid sample is transparent while the solid sample is not, visual observation of the red mark indicates that at this point the sample is in liquid phase. The 

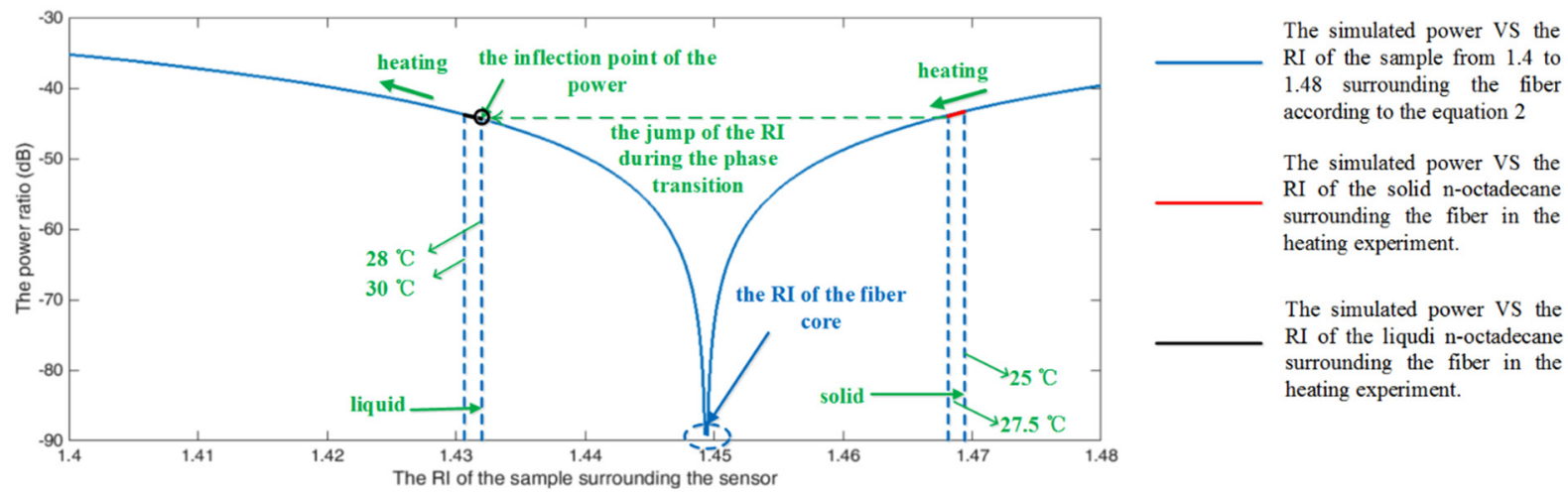

Figure 2. Simulated relationship between the reflected power ratio and the refractive index of the surrounding the probe material sample.

temperature in the vicinity of the fiber probe was monitored with a K-type thermocouple with an error of $\pm 0.004 \times T$, where $T$ is the actual temperature. The thermocouple was fixed close to the probe. A Peltier element with the rated voltage of $12 \mathrm{~V}$ was used as a thermoelectric heater/cooler, placed on the top of the glass cylindrical container, to eliminate the effect of thermal convection.

To reduce the effect caused by the instability of the light source, a ratiometric measurement scheme is used in the experiments [12]. The power ratio at the detector is measured as the ratio of power at Channel 1 and Channel 2 of the power meter and is described as [16]

$$
P=10\{\lg [R(I+x)]-\lg (I+x)\}=10 \lg R
$$

where $I$ is one half of the input power of the optical source (one arm of the $3 \mathrm{~dB}$ coupler), $x$ is the power fluctuation of the optical source, $R$ is the reflectivity of the fiber probe which can be described using the well-known Fresnel equation:

$$
R=\left(\frac{n_{1}-n_{2}}{n_{1}+n_{2}}\right)^{2}
$$

where $n_{1}$ is the RI of the single-mode fiber core (assumed equal to 1.4494 at wavelength of $1550 \mathrm{~nm}$ ) and $n_{2}$ is the RI of the material surrounding the sensor.

The simulated relationship between the reflected power ratio and the RI of the surrounding material of the sensor probe was calculated using equations (1) and (2), and the result is shown using the blue line in figure 2. As one can see from figure 2, for all $n_{2}$ values (the RI of the sample under test) smaller than $n_{1}$ (the RI of the fiber core), the reflected power ratio decreases monotonically with the increase of $n_{2}$ until the latter becomes equal to $n_{1}$. For $n_{2}$ values greater than $n_{1}$ the reflected power ratio increases monotonically with the increase of the surrounding RI $\left(n_{2}\right)$.

For a phase change material it is possible to redraw figure 2, based on the known RI behavior of n-octadecane with changes in temperature.

The RI of n-octadecane decreases with an increase in temperature. In its solid phase it experiences a progressive uninterrupted decrease in RI with an increase in temperature, but as soon as the n-octadecane becomes liquid, its RI decreases sharply from circa 1.468 to 1.432 [15]. Based on these approximate values for the RI of n-octadecane and its temperature dependence, the behaviour of the optical power ratio reflected by the probe can be interpreted as follows in accordance with the model presented in figure 2 .

In the experiment, the temperature range chosen for the n-octadecane is from $25^{\circ} \mathrm{C}$ to $30{ }^{\circ} \mathrm{C}$, with the phase change occurring at approximately $28{ }^{\circ} \mathrm{C}$. Since the solid n-octadecane has an RI that is larger than that of the fiber core, any increase in temperature results in a decrease of the material's $\mathrm{RI}$ and in the reflected power ratio $\left(n_{2} \geqslant 1.4494\right.$, the core RI, in figure 2). This is illustrated with the red line in figure 2 , for a temperature increase from $25^{\circ} \mathrm{C}$ to $27.5^{\circ} \mathrm{C}$. As soon as the n-octadecane sample melts to form a liquid, its RI undergoes a discontinuous decrease so it is lower than that of the fiber core. From that point, any further increase in temperature causes a decrease in the RI value once more but now because the RI is lower than that of the fiber core, in accordance with equation (2) and the blue trace in figure 2 , the reflected power ratio now increases (region $n_{2} \leqslant 1.4494$, the core RI, in figure 2), which is illustrated as the black line shown in figure 2 for a temperature change from $28^{\circ} \mathrm{C}$ to $30^{\circ} \mathrm{C}$.

Not only therefore is there a discontinuous jump in the RI as temperature increases through the phase change temperature region, there is also a change in the slope of the power ratio versus RI characteristic for the n-octadecane as it changes to a different phase state, as witnessed by the different slopes of the red line compared to the black line in figure 2. Assuming one is observing the reflected power ratio as the temperature increases, then there is in effect an inflection point evident in the reflected power ratio as the temperature increases. This inflection point in the reflected power ratio is the key to determining the occurrence of the phase change in the vicinity of the fiber probe. For brevity the explanation above is provided for the heating case only, but there is an analogous behaviour for the case of cooling, where there is again an inflection point, so that phase change can be also detected by finding the inflection point of the reflected power ratio as the temperature decreases with cooling.

Finally it should be noted that the RI values used above for n-octadecane are known to be approximately 1.468 for solid and 1.432 of liquid phases at the wavelength of $600 \mathrm{~nm}$ [15]. Since the experimental setup used here operated circa $1550 \mathrm{~nm}$, this RI data from [15] and the Cauchy's equation [17] was used to calculate the approximate values of 


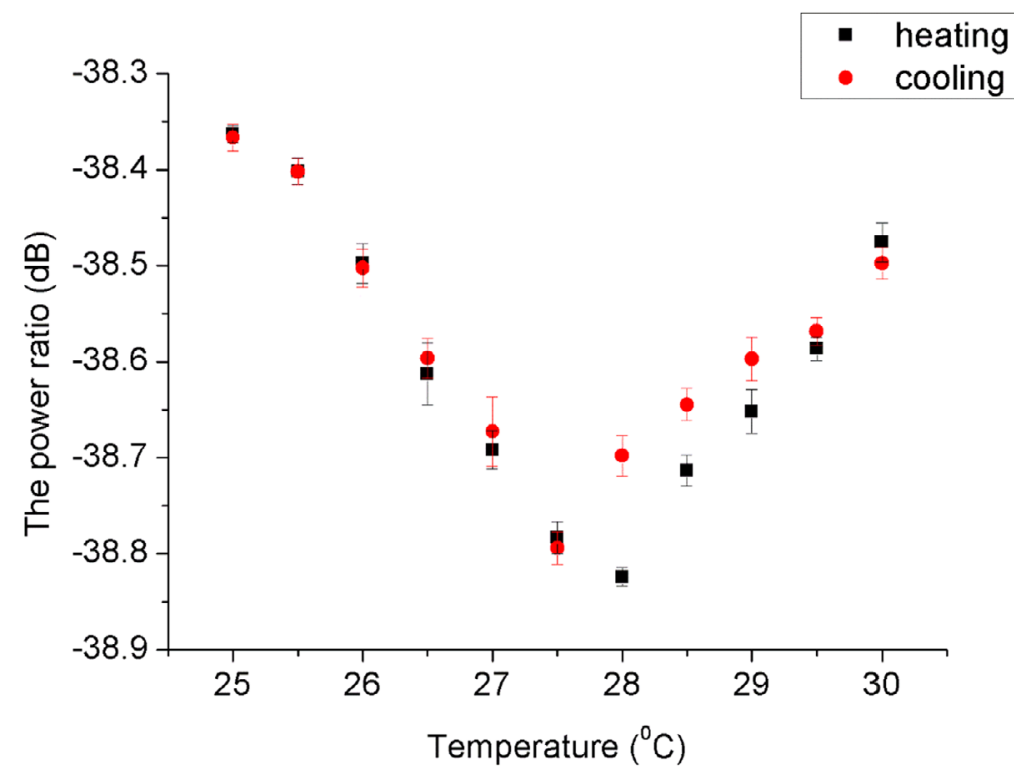

Figure 3. Reflected power ratio measured at different temperatures during the heating and the cooling cycle of the n-octadecane sample: inflection point at $\sim 28^{\circ} \mathrm{C}$ in the heating corresponds to the solid-liquid phase change and at $\sim 27.5^{\circ} \mathrm{C}$ corresponds to the liquid-solid phase change in the cooling.

the RI of the n-octadecane at $1550 \mathrm{~nm}$. The results of the calculations showed that the RI of n-octadecane a maximum decrease of $0.1 \%$ for the solid and $0.08 \%$ for the liquid phase over the wavelength range from 1200 to $1600 \mathrm{~nm}$. Based on such very small variations, the influence of wavelength on the RI can be effectively ignored.

\section{Experimental results and discussion}

In order to experimentally demonstrate the operation of the proposed sensor, a series of heating and cooling experiments were carried out for a $25 \mathrm{ml}$ sample of n-octadecane with the purity of $99 \%$ purity (Sigma Aldrich), using the experimental setup shown in figure 1 . To eliminate the effects of thermal convection, the Peltier element used for heating and cooling of the sample was placed at the top of the container. The fiber probe and the thermocouple where inserted into the container through the bottom, and the distance between the fiber probe and the thermocouple was approximately $2 \mathrm{~mm}$. The thermocouple was connected to a logger for recording the temperature of the n-octadecane in close proximity of the fiber sensor probe. To ensure the stability of the surrounding temperature, the experimental setup was placed inside a transparent heat insulating enclosure. A photo camera was placed inside the transparent heat insulating enclosure and connected with a PC. The camera shutter was controlled by the PC. The photo images were taken at the same time as the reflected power ratio and temperature data were recorded with steps of $0.5^{\circ} \mathrm{C}$.

Figure 3 illustrates the dependence of the measured reflected power ratio of the sensor probe versus temperature of n-octadecane during its heating and cooling cycle. The heating experiment was carried by setting the temperature of Peltier element to $50{ }^{\circ} \mathrm{C}$ when the material sample was at room temperature $\left(20^{\circ} \mathrm{C}\right)$. Every data point in figure 3 is an average of five different heating experiments. The average error is
$0.017 \mathrm{~dB}$. We believe that the most likely source of error is due to the system noise itself as we have measured power variation of $0.016 \mathrm{~dB}$ when the fiber sensor was immersed into the n-octadecane at a fixed room temperature. The other possible reason of the error is the changing mechanical forces acting upon the fiber during the phase change, resulting in slight movement of the probe caused by mechanical stress.

As can be seen from the figure in the heating experiment the reflected power ratio changes from $-38.36 \mathrm{~dB}$ to $-38.78 \mathrm{~dB}$ in the range of temperatures from $25^{\circ} \mathrm{C}$ to $27.5^{\circ} \mathrm{C}$, confirming that heating of n-octadecane in the solid phase causes its RI to decrease, resulting in the decrease of the reflected power ratio. Comparison of the experimentally measured reflected power ratio values with the calculated results (shown in figure 2) leads to the corresponding estimated RI change of solid n-octadecane from 1.4783 to 1.4769 assuming there is no absorption by the of n-octadecane. In the vicinity of $28^{\circ} \mathrm{C}$ n-octadecane becomes liquid, which causes the change in the power ratio dependence as predicted by the proposed physical model. In the temperature range from $28{ }^{\circ} \mathrm{C}$ to $30{ }^{\circ} \mathrm{C}$, the measured reflected power ratio changes from $-38.82 \mathrm{~dB}$ to $-38.48 \mathrm{~dB}$. During the heating in its liquid phase the RI of n-octadecane continues to decrease, however, in this case the RI values of n-octadecane become smaller than RI of the silica fiber, resulting in the increase of the reflected back power ratio as predicted by the simulations in figure 2 , where a change in the power ratio from -38.82 to $-38.48 \mathrm{~dB}$ corresponds to the change in the liquid RI from 1.4226 to 1.4215 .

It should be noted that in our experiment the RI of n-octadecane is slightly different from the value reported in the [15] because of the absorption loss of the n-octadecane, and the differences in the material purity and the temperature, which is caused by the error, for example, the distance of the thermocouple and the probe. Figure 4 shows images of the n-octadecane sample taken at different temperatures. It 

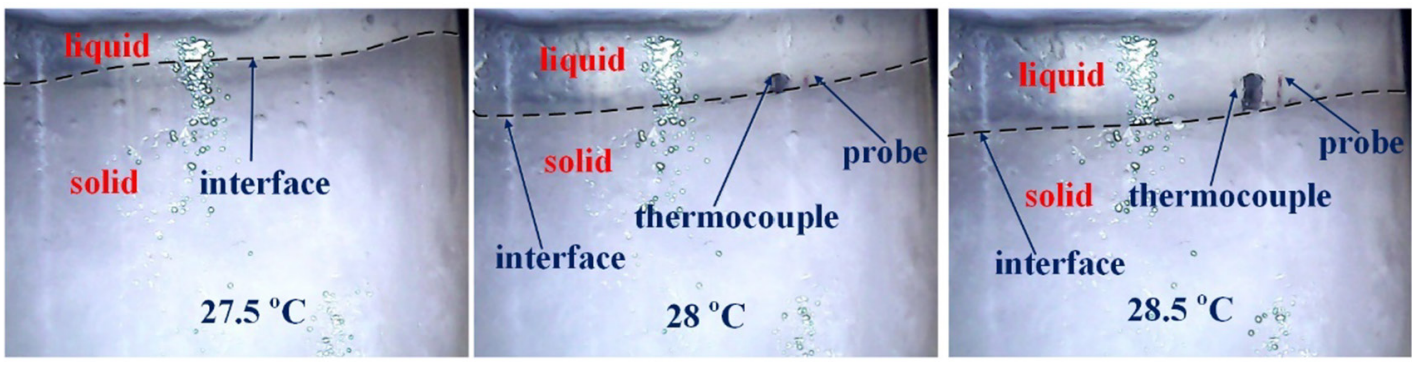

Figure 4. Photographs of the solid-liquid interface position at temperatures: at $28{ }^{\circ} \mathrm{C}$ both the thermocouple and fiber optic probe tips are clearly visible (the image corresponds to the inflection point in figure 3 ).
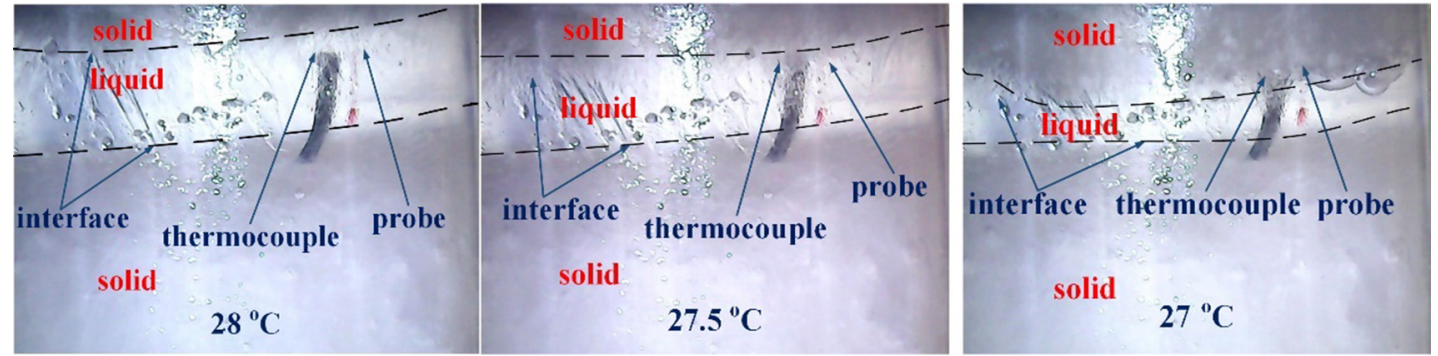

Figure 5. Photographs of the solid-liquid interface position at different temperatures: at $27.5^{\circ} \mathrm{C}$ both the thermocouple and fiber optic probe tips are in contact with the solid surface (the image corresponds to the inflection point in figure 3 ).

can be seen therefore, that the point of inflection within the experimental graph corresponds to the solid-to-liquid phase transition of n-octadecane demonstrating that monitoring of the reflected power ratio change in time (or with temperature) and determining the point of inflection of the reflected power ratio dependence allows for an accurate detection of the phase change of the material at the point location of the sensor probe. It should be noted that the if the proposed method is used for detecting the phase change, the temperature information is not needed, and it is capable of providing information regarding the phase state at a particular location within a volume of material in real time.

For the cooling experiment the temperature of Peltier element was set to $20{ }^{\circ} \mathrm{C}$, while the material sample was in liquid phase at $30{ }^{\circ} \mathrm{C}$. Cooling of the n-octadecane sample causes increase in its RI. The reflected power ratio changes from $-38.49 \mathrm{~dB}$ to $-38.79 \mathrm{~dB}$ as the temperature decreases from $30{ }^{\circ} \mathrm{C}$ to $27.5^{\circ} \mathrm{C}$. In accordance with our previous simulations, the RI in this temperature interval changes from 1.4216 to 1.4225 . At $27{ }^{\circ} \mathrm{C}$ the n-octadecane becomes solid which causes the sudden increase in the reflected power ratio up to $-38.67 \mathrm{~dB}$, followed by its subsequent increase to $-38.37 \mathrm{~dB}$. During the cooling in the solid phase, the RI increases but since the RI of the solid n-octadecane is larger than the RI of the fiber core, the power ratio increases as predicted by the simulation, where a change in the power ratio from $-38.67 \mathrm{~dB}$ to $-38.37 \mathrm{~dB}$ corresponds to the change in the solid RI from 1.4772 to 1.4782 . The freezing point is lower than the melting point due to the hysteresis phenomenon. The average error is $0.019 \mathrm{~dB}$. The possible source of the error is the same as the one in the heating experiment. As it can be seen from figure 3 , the inflection of the reflected power ratio dependence corresponds to the phase change point for the n-octadecane sample. Figure 5 illustrates the gradual shift
Table 1. The relationship between the material's phase and the slope of the power ratio.

\begin{tabular}{lll}
\hline & Solid phase & Liquid phase \\
\hline Heating & $\Delta P<0$ & $\Delta P>0$ \\
Cooling & $\Delta P>0$ & $\Delta P<0$ \\
\hline
\end{tabular}

of the interface between liquid and solid phases during the cooling experiment. It can be seen that during cooling the point of inflection within the experimental graph corresponds to the image where solid-liquid interface reaches the fiber probe, confirming the phase change at the location of the probe.

Moreover, one can determine whether the n-octadecane is in solid or liquid phase from the slope of the power ratio $(\Delta P)$ as shown in table 1. During the heating process, if the slope is negative, then the sample is solid, while if the slope is positive, the sample is liquid. During the cooling process, if the slope is positive, the sample is solid, if the slope is negative, the sample is liquid. The sensor can thus detect the phase change at a certain point within the sample by comparing two consecutive reflected power ratio measurements during the material heating or cooling cycles. Moreover, if supercooling occurs, the temperature will be lower than solidification temperature measured by the thermocouple, and the phase of the sample will be detected as liquid phase by the sensor, so the supercooling can be detected.

\section{Conclusion}

In conclusion, a novel method for in situ detection of the solidliquid and liquid-solid phase changes in n-octadecane, based on Fresnel reflection in a fiber sensor probe has been proposed and demonstrated experimentally. From the experimental results it can be seen that the melting point is approximately 
at $28^{\circ} \mathrm{C}$ and the freezing point is at $27.5^{\circ} \mathrm{C}$. The accuracy of the phase change detection was confirmed by visual means. The proposed method allows for a simple, low cost, accurate and reliable in situ detection of liquid-solid and solid-liquid phase changes in n-octadecane without the need for temperature measurements. Moreover, at the same time supercooling of PCMs can be detected which is a critical issue for optimization of and control of latent heat thermal energy storage technologies. This point-sensing technique can be expanded by increasing the number of sensor probes and applied for studies and optimization of heat distribution within the PCM material employed in energy storage systems. The results of this work suggest that such a simple optical fiber Fresnel reflection sensor can be used for detection of liquid-solid phase changes in other materials with similar to n-octadecane thermo-optic properties. The proposed method allows for a simple, low cost, accurate and reliable in situ detection of liquid-solid and solid-liquid phase changes in n-octadecane without the need for temperature measurements. This pointsensing technique can be expanded by increasing a number of sensor probes and applied for studies and optimization of heat distribution within the PCM material employed in energy storage systems. The results of this work suggest that such a simple optical fiber sensor can be used for detection of liquidsolid phase changes in other materials with similar to n-octadecane thermo-optic properties.

\section{ORCID iDs}

Wei Han (1) https://orcid.org/0000-0002-6675-4775

Qiang Wu (ㄱ) https://orcid.org/0000-0002-2901-7434

\section{References}

[1] Pielichowska K and Pielichowski K 2014 Phase change materials for thermal energy storage Prog. Mater. Sci. 65 67-123

[2] Anderson R L, Adams R K and Duggins B C 1979 Limitations of Thermocouples in Temperature Measurements Oak Ridge National Lab Report CONF-790505-14 (Oak Ridge, TN: Oak Ridge National Laboratory)

[3] Akamatsu S, Faivre G and Ihle T 1995 Symmetry-broken double fingers and seaweed patterns in thin-film directional solidification of a nonfaceted cubic crystal Phys. Rev. E 514751

[4] Yin H and Koster J N 1999 In situ observation of concentrational stratification and solid-liquid interface morphology during $\mathrm{Ga}-5 \%$ in alloy melt solidification J. Cryst. Growth 205 590-606

[5] Shirtcliffe T G L, Huppert H E and Worster M G 1991 Measurement of the solid fraction in the crystallization of a binary melt J. Cryst. Growth 113 566-74

[6] Lazaro A, Peñalosa C, Solé A, Diarce G, Haussmann T, Fois M, Zalba B, Gshwander S and Cabeza L F 2012 Intercomparative tests on phase change materials characterization with differential scanning calorimeter Appl. Energy 109 415-20

[7] Zhao Y, Cai L and Li X G 2017 In-fiber modal interferometer for simultaneous measurement of curvature and temperature based on hollow core fiber Opt. Laser Technol. 92 138-41

[8] Liu Y, Lang C, Wei X and Qu S 2017 Strain force sensor with ultra-high sensitivity based on fiber inline Fabry-Perot micro-cavity plugged by cantilever taper Opt. Express 25 7797-806

[9] Nguyen L V, Hwang D, Moon S, Moon D S and Chung Y 2008 High temperature fiber sensor with high sensitivity based on core diameter mismatch Opt. Express 16 11369-75

[10] Millo A, Raichlin Y and Katzir A 2005 Mid-infrared fiber-optic attenuated total reflection spectroscopy of the solid-liquid phase transition of water Appl. Spectrosc. 59 460-6

[11] Quan C, Later K, Yang L, Peng S, Zhang A, Hao Q, Zhang J, Sun W, Yuan L and Peng G D 2012 FBG application in monitoring the liquid-solid and gas-liquid phase transitions of water 3rd Asia Pacific Optical Sensors Conf. vol 8351 p 835123

[12] Mani P, Rallapalli A, Machavaram V R and Sivaramakrishna A 2016 Monitoring phase changes in supercooled aqueous solutions using an optical fiber Fresnel reflection sensor Opt. Express 24 5395-410

[13] Choi M K 2016 Using paraffin PCM to make optical communication type of payloads thermally self-sufficient for operation in Orion crew module 14th Int. Energy Conversion Engineering Conf. p 4601

[14] Mondal S 2008 Phase change materials for smart textiles-an overview Appl. Therm. Eng. 28 1536-50

[15] Kim M S, Kim M K, Jo S E, Joo C and Kim Y J 2016 Refraction-assisted solar thermoelectric generator based on phase-change lens Sci. Rep. 627913

[16] Hatta A M, Farrell G, Wang Q, Rajan G, Wang P and Semenova Y 2008 Ratiometric wavelength monitor based on singlemode-multimode-singlemode fiber structure Microw. Opt. Technol. Lett. 50 3036-9

[17] Jenkins F A and White H E 1937 Fundamentals of Optics (New York: McGraw-Hill) 